\title{
Hyperbolic Plateau problems
}

\author{
Graham Smith \\ 23rd May 2011 \\ Centre de Recerca Matemàtica, \\ Facultat de Ciències, Edifici C, \\ Universitat Autònoma de Barcelona, \\ 08193 Bellaterra, \\ Barcelona, \\ SPAIN
}

\begin{abstract}
We consider surfaces of constant Gaussian curvature immersed in 3-dimensional manifolds, and we strengthen the compactness result of Labourie in the case where the ambient manifold is 3-dimensional hyperbolic space. This allows us to prove results of existence of solutions to the asymptotic Plateau problem, as defined by Labourie, and the continuous dependence of these solutions on the data.
\end{abstract}

Key Words: Plateau problem, Minkowski problem, Gaussian curvature, hyperbolic space

AMS Subject Classification: 32Q65, 51M10, 53C42, 53C45, 53D10, 58D10, 58J05 


\section{Hyperbolic Plateau problems}

\section{1 - Introduction.}

The Gaussian curvature of an immersed surface has interested differential geometers right from the very beginning with the announcement of Gauss' famous Teorema Egregium, [4]. Having established this concept, it is natural to ask when there exist surfaces of constant Gaussian curvature in a given manifold subject to certain prescribed conditions which are typically geometric or topological in nature. For example, in the compact case, the Plateau problem and its various variants ask for compact immersed surfaces of constant Gaussian curvature with prescribed boundary (c.f. [3], [6], [13], [18] etc.). In the non-compact case, Minkowski type problems ask for complete immersed hypersurfaces of constant Gaussian curvature whose normal vector field is prescribed by some sort of Gauss map (c.f. [8], [9], [12] etc.).

On closer examination, PDE considerations show that the qualitative nature of the problem is foremost affected by the sign of the Gaussian curvature. Indeed, the resulting PDE is elliptic, hyperbolic, or totally degenerate depending on whether the Gaussian curvature is positive, negative or zero respectively. Consequently, in the first case, the highly developed theory of regularity and compactness results for elliptic PDEs translates into a stronger geometric theory than can in general be obtained in the remaining cases. In the current setting, we go even further, as Labourie shows in [9] a straightforward but remarkable relationship between surfaces of constant positive Gaussian curvature on the one hand, and pseudo-holomorphic curves in the tangent bundle of the ambient manifold on the other which, through the powerful techniques developed by Gromov in [5], readily yields a strong compactness result (Theorem 3.3) requiring weak hypotheses and involving a unique, elementary mode of degeneration which itself may often be excluded through straightforward geometric considerations. Using the continuity method and various limiting processes, this in turn yields existence results for solutions to the Plateau and Minkowksi problems (c.f. $[9]$ and $[17])$.

By definition, any compactness result assumes a choice of topology, and in the case of Labourie's compactness theorem (Theorem 3.3), the topology in question is the $C^{\infty}$ pointed Cheeger/Gromov topology on the space of complete, immersed surfaces (i.e. the topology of smooth convergence modulo reparametrisation). This topology is too weak to provide much control over the structure of the limit submanifolds. For example, the limit depends on the preferred points chosen, and in the case of surfaces, although on the one hand the genus is lower semi-continuous, the number of singularities is not even semi-continuous, compactness is not a closed property, and so on. As a consequence, it is rare that convergence in this topology is sufficient for any application, and supplementary techniques are often necessary in order to actually prove results. It is for this reason that in this paper, restricting attention to the case where the ambient manifold is 3-dimensional hyperbolic space, we deduce a much stronger compactness theorem, which in turn permits us to recover a special case of the existence result of [16] as well as to show the continuous dependence of the solutions, which are unique, upon the data.

Before stating our results, it is worth observing how the study of complete hypersurfaces of positive Gaussian curvature in $\mathbb{H}^{3}$ further subdivides into three different cases, depending 


\section{Hyperbolic Plateau problems}

on the value, $k$, of the curvature. When $k>1$, the intrinsic geometry is spherical, and we readily show that the immersion is a geodesic sphere of radius determined by $k$. When $k=1$, the intrinsic geometry of the surface is Euclidean, and the immersion is either a horosphere, or a cylinder of points lying at constant distance from a complete geodesic. It is thus only in the case where $k \in] 0,1$, and the intrinsic geometry is hyperbolic, that a rich zoology arises, and it is for this reason that we restrict attention henceforth to these values of $k$ (note, however, that by admitting singularities nonetheless we recover an interesting zoology also in the preceding two cases, as in for example [11]).

We now describe the setting in which our results are proven. Let $\mathbb{H}^{3}$ be 3 -dimensional hyperbolic space. Let $T \mathbb{H}^{3}$ be the tangent bundle over $\mathbb{H}^{3}$ and let $U \mathbb{H}^{3}$ be the circle bundle of vectors of unit length in $T \mathbb{H}^{3}$. Let $S$ be an oriented surface, let $i: S \rightarrow \mathbb{H}^{3}$ be an immersion, and let $\mathrm{N}_{i}: S \rightarrow \mathbb{H}^{3}$ be the unit normal vector field over $i$ compatible with the orientation. We define the Gauss lift $\hat{\imath}: S \rightarrow U \mathbb{H}^{3}$ by:

$$
\hat{\imath}=\mathrm{N}_{i}
$$

\section{Definition 1.1}

For $k \in] 0,1$, we say that the immersed hypersurface $\Sigma:=(S, i)$ is a $k$-surface if and only if:

(i) $i$ is everywhere locally strictly convex and is of constant Gaussian curvature equal to $k$; and

(ii) $\hat{\imath}$ is complete.

Remark: The condition that the Gauss lift rather than the immersion itself be complete, although apparently strange, is natural from the perspective of Labourie's compactness theorem which, strictly speaking, operates entirely within the unitary bundle (c.f. [9]). This often leads to subtle complexities in the geometric arguments required. However, in the case of most interest to us, being that of solutions generated by ramified coverings of the sphere, completeness of $i$ follows from completeness of $\hat{\imath}$ (c.f. [15]).

Let $\partial_{\infty} \mathbb{H}^{3}$ be the ideal boundary of $\mathbb{H}^{3}$ (c.f. [1]). We define the Gauss-Minkowksi map, $\vec{n}: U \mathbb{H}^{3} \rightarrow \partial_{\infty} \mathbb{H}^{3}$ as follows: choose $V \in U \mathbb{H}^{3}$, and define $\vec{n}(V)$ to be the point in $\partial_{\infty} \mathbb{H}^{3}$ towards which $V$ points. Explicitly, if $\gamma: \mathbb{R} \rightarrow \mathbb{H}^{3}$ is the unique geodesic such that:

$$
\left(\partial_{t} \gamma\right)(0)=V
$$

then:

$$
\vec{n}(V)=\gamma(+\infty)=\operatorname{Lim}_{t \rightarrow+\infty} \gamma(t)
$$

We recall that $\partial_{\infty} \mathbb{H}^{3}$ has the conformal structure of the Riemann sphere. Following [9], we make the following definition: 


\section{Definition 1.2}

An asymptotic Plateau problem is a pair $(S, \varphi)$ where $S$ is a Riemann surface, and $\varphi: S \rightarrow \mathbb{H}^{3}$ is a locally conformal mapping.

Given an asymptotic Plateau problem, $(S, \varphi)$, and given $k \in] 0,1[$, the smooth immersion $i: S \rightarrow \mathbb{H}^{3}$ is said to be a solution to $(S, \varphi)$ if and only if:

(i) $(S, i)$ is a $k$-surface; and

(ii) $\varphi=\vec{n} \circ i$.

Remark: This definition superficially appears more restrictive than that given in [9], where $S$ may be any topological surface and $\varphi$ a local homeomorphism. However, pulling back the conformal structure of $\partial_{\infty} \mathbb{H}^{3}$ through $\varphi$ readily transforms an asymptotic Plateau problem in the sense of [9] into an asymptotic Plateau problem in the current sense, and so we see that no generality is lost.

Remark: we see that an asymptotic Plateau problem is in fact closer to the Minkowski problem than the Plateau problem. We nonetheless retain the current terminology for consistency with [9] and [15].

The main result of this paper is the following Theorem which follows immediately from Proposition 5.3:

\section{Theorem 1.3, Compactness}

Let $\mathbb{D}$ be the Poincaré disc. Let $\left(\varphi_{n}\right)_{n \in \mathbb{N}}: \mathbb{D} \rightarrow \partial_{\infty} \mathbb{H}^{3}$ be locally conformal maps. Choose $k \in] 0,1\left[\right.$ and for all $n$ let $i_{n}: \mathbb{D} \rightarrow \mathbb{H}^{3}$ be a $k$-surface which is a solution to the asymptotic Plateau problem $\left(\mathbb{D}, \varphi_{n}\right)$. Suppose that there exists a locally conformal map $\varphi_{0}: \mathbb{D} \rightarrow \partial_{\infty} \mathbb{H}^{3}$ towards which $\left(\varphi_{n}\right)_{n \in \mathbb{N}}$ converges in the local uniform sense. Then there exists an immersion $i_{0}: \mathbb{D} \rightarrow \mathbb{H}^{3}$ such that

(i) $\left(\mathbb{D}, i_{0}\right)$ is a $k$-surface;

(ii) $i_{0}$ is a solution to the asymptotic Plateau problem $\left(\mathbb{D}, \varphi_{0}\right)$; and

(ii) $\left(i_{n}\right)_{n \in \mathbb{N}}$ converges to $i_{0}$ in the $C_{\mathrm{loc}}^{\infty}$ sense.

This yields a short, alternative proof of the following special case of Theorem 1.1 of [16]:

Theorem 1.4, Existence

Let $\mathbb{D}$ be the Poincaré disc. Let $\varphi: \mathbb{D} \rightarrow \partial_{\infty} \mathbb{H}^{3}$ be a locally conformal map. For all $k \in] 0,1\left[\right.$, there exists a unique immersion $i: \mathbb{D} \rightarrow \mathbb{H}^{3}$ such that:

(i) $(\mathbb{D}, i)$ is a $k$-surface; and

(ii) $i$ is a solution to the asymptotic Plateau problem $(\mathbb{D}, \varphi)$. 


\section{Hyperbolic Plateau problems}

Moreover the following continuity result, being valid also in the non-cocompact case, is thus stronger than what may be obtained via the techniques of [16]:

\section{Theorem 1.5, Continuous Dependence}

Let $\mathbb{D}$ be the Poincaré disc. Let $\left(\varphi_{n}\right)_{n \in \mathbb{N}}, \varphi_{0}: \mathbb{D} \rightarrow \partial_{\infty} \mathbb{H}^{3}$ be locally conformal maps. Choose $k \in] 0,1\left[\right.$, and for all $n \in \mathbb{N} \cup\{0\}$, let $i_{n}: \mathbb{D} \rightarrow \mathbb{H}^{3}$ be the unique immersion such that:

(i) $\left(\mathbb{D}, i_{n}\right)$ is a $k$-surface; and

(ii) $i_{n}$ is the unique solution to the asymptotic Plateau problem $(\mathbb{D}, \varphi)$.

Suppose that $\left(\varphi_{n}\right)_{n \in \mathbb{N}}$ converges to $\varphi_{0}$ in the local uniform sense, then $\left(i_{n}\right)_{n \in \mathbb{N}}$ converges to $i_{0}$ in the $C_{\text {loc }}^{\infty}$ sense.

This paper is a greatly revised version of the second chapter of the author's doctoral thesis. The author would like to thank François Labourie for having proposed this problem, for his guidance during that period, and for his encouragement to prepare the current version. The author would also like to thank the Université Paris XI, and the Max Planck Insitute for Mathematics in the Sciences in Leipzig for providing the conditions required to prepare the previous version of this paper. The author would like thank the Centre de Recerca Matemàtica in Barcelona for providing the conditions required to prepare the current version of this paper which was written whilst the author was benifitting from a Marie Curie Postdoctoral fellowship.

\section{2 - Conformal Structure.}

For $r>0$, let $S_{r} \mathbb{H}^{3}$ be the bundle of spheres of radius $r$ in $T \mathbb{H}^{3}$. Let $S$ be an oriented surface and let $i: S \rightarrow \mathbb{H}^{3}$ be an immersion. Let $\mathrm{N}_{i}$ be the unit normal vector field over $i$ compatible with the orientation. We define $\hat{\imath}_{r}: S \rightarrow S_{r} \mathbb{H}^{3}$ by:

$$
\hat{\imath}_{r}=r \mathrm{~N}_{i} \text {. }
$$

We call $\hat{\imath}_{r}$ the Gauss lift of $i$ at height $r$. Let $\hat{g}$ be the Sasaki metric over $T \mathbb{H}^{3}$ (c.f. [14]). Let $I_{i}$ and $I I I_{i}$ be the first and third fundamental forms of $i$. We readily see that:

$$
\hat{\imath}_{r}^{*} \hat{g}=I_{i}+r^{2} I I I_{i}
$$

Let $J_{i, r}$ be the complex structure compatible with the orientation induced over $\Sigma$ by the metric $\hat{\imath}_{r}^{*} \hat{g}$. We henceforth refer to $J_{i, r}$ as the complex structure induced by $\hat{\imath}_{r}$. We shall see that this complex structure plays a central role in the sequel.

Let $\partial_{\infty} \mathbb{H}^{3}$ be the ideal boundary of $\mathbb{H}^{3}$. We define the Gauss-Minkowski mapping $\vec{n}: S_{r} \mathbb{H}^{3} \rightarrow \partial_{\infty} \mathbb{H}^{3}$ as in the introduction. Given an immersion $i: S \rightarrow \mathbb{H}^{3}$, the composition $\left(\vec{n} \circ \hat{\imath}_{i, r}\right.$ ) (which is indepedant of $r$ ) is everywhere an orientation preserving local diffeomorphism between the Riemann surfaces $\left(S, J_{i, r}\right)$ and $\partial_{\infty} \mathbb{H}^{3}=\hat{\mathbb{C}}$. In general, this map is not conformal but is quasiconformal. We thus recall the definition of quasiconformality. Let $U \subseteq \mathbb{C}$ be open and let $\alpha: U \rightarrow \mathbb{C}$ be a smooth, orientation preserving 


\section{Hyperbolic Plateau problems}

mapping which is everywhere a local diffeomorphism. For all $z \in U$, the derivative of $\alpha$ is given by:

$$
D \alpha_{z} \cdot w=\partial \alpha(z) w+\bar{\partial} \alpha(z) \bar{w} .
$$

We define $\mu(\alpha)$, the complex dilatation of $\alpha$, by:

$$
\mu(\alpha)(z)=\frac{\bar{\partial} \alpha(z)}{\partial \alpha(z)}
$$

For $K \geqslant 1$, we say that $\alpha$ is $K$-quasiconformal if and only if for all $z \in U$ :

$$
|\mu(\alpha)(z)| \leqslant \frac{K-1}{K+1}
$$

We recall that the concept of $K$-quasiconformality readily extends to orientation preserving local homeomorphisms, and that a local homeomorphism is 1-quasiconformal if and only if it is smooth and conformal (c.f. [7]).

\section{Lemma 2.1}

Choose $k \in] 0,1\left[\right.$ and let $r=k^{-1 / 2}>1$. Let $i: \Sigma \rightarrow \mathbb{H}^{3}$ be a locally strictly convex immersion, let $\hat{\imath}_{r}: S \rightarrow S_{r} \mathbb{H}^{3}$ be the Gauss lift of $i$ at height $r$, and let $J_{i, r}$ be the complex structure induced over $S$ by $\hat{\imath}_{r}$. If $i$ has constant Gaussian curvature equal to $k$, then $\left(\vec{n} \circ \hat{\imath}_{r}\right):\left(S, J_{i, r}\right) \rightarrow \partial_{\infty} \mathbb{H}^{3}$ is $r$-quasiconformal.

Proof: We identify $\mathbb{H}^{3}$ with $H^{+}$, the upper half space in $\mathbb{R}^{3}$. We identify $S_{r} \mathbb{H}^{3}$ with $H^{+} \times S^{2}$. Using elementary Euclidean geometry, we readily show:

$$
\vec{n}((x, y, z),(u, v, w))=(x, y)+\frac{z}{1-w}(u, v)
$$

Choose $p_{0} \in S$. By applying an isometry of $\mathbb{H}^{3}$, we may assume that there exists a neighbourhood, $U$, of 0 in $\mathbb{R}^{2}$, a neighbourhood, $V$, of $p_{0}$ in $S$ and a smooth function $\left.f: \mathbb{R}^{2} \rightarrow\right] 0, \infty$ [ such that $i(V)$ coincides with the graph of $f$ over $U$. We may suppose, moreover, that $f(0)=1$ and $D f(0)=0$. Since the result is invariant under reparametrisation, it suffices to prove it for $\hat{f}: U \rightarrow \mathbb{H}^{3}$ given by:

$$
\hat{f}(x, y)=(x, y, f(x, y))
$$

Let $\left(e_{1}, e_{2}\right)$ be an orthonormal basis of $\mathbb{R}^{2}$ with respect to which $\operatorname{Hess}_{0}(f)$ is diagonal, and let $\left(\lambda_{1}, \lambda_{2}\right)$ be the corresponding eigenvalues. Let $\mathrm{N}_{\hat{f}}$ be the unit normal vector field over $\hat{f}$ compatible with the orientation. We may assume that $\mathbf{N}_{\hat{f}}$ points downwards. Since the hyperbolic metric over $H^{+}$is conformally equivalent to the Euclidean metric, $\mathrm{N}_{\hat{f}}$ coincides up to a scaling factor with the Euclidean unit normal, and we readily obtain:

$$
\mathrm{N}_{\hat{f}}(x, y)=\left((x, y, f(x, y)),\left(1+\|D f\|^{2}\right)^{-1}\left(\partial_{x} f, \partial_{y} f,-1\right)\right)
$$




\section{Hyperbolic Plateau problems}

Bearing in mind that $\hat{f}(0)=(0,0,0)$ and $D f(0)=(0,0)$, using the chain rule, we readily obtain:

$$
D\left(\vec{n} \circ \mathrm{N}_{\hat{f}}\right)(0)=M:=\operatorname{Id}+\frac{1}{2}\left(\begin{array}{ll}
\lambda_{1} & 0 \\
0 & \lambda_{2}
\end{array}\right) .
$$

Let $I_{0}(f), I I_{0}(f)$ and $I I I_{0}(f)$ be the first, second and third fundamental forms of $\hat{f}$ with respect to the hyperbolic metric at 0 . Since $f(0)=1$ and $D f(0)=0$, we readily see that $I$ coincides with the Euclidean metric over $\mathbb{R}^{2}$. Let $I I_{0}^{e}(f)$ be the second fundamental form of $\hat{f}$ with respect to the Euclidean metric at 0 . Observe that $I I_{0}(f)-I I_{0}^{e}(f)$ only depends on $\mathrm{N}_{\hat{f}}(0)$. Let $\left.g: U \rightarrow\right] 0, \infty$ [ be the function whose graph is a portion of the unit sphere in $\mathbb{R}^{3}$ centred at 0 . In particular, $g(0)=1$ and $D g(0)=0$. We choose $\mathbf{N}_{\hat{g}}(0)$ to be the downward pointing normal. Then, by definition:

$$
I I_{0}^{e}(g)\left(e_{i}, e_{j}\right)=-\delta_{i j},
$$

However, the graph of $g$ is a totally geodesic subspace of $\mathbb{H}^{3}$. Thus:

$$
I I_{0}(g)=0 .
$$

Since $\mathrm{N}_{\hat{f}}$ points downwards, we readily obtain:

$$
I I_{0}^{e}(f)\left(e_{i}, e_{j}\right)=\operatorname{Hess}_{0}(f)\left(e_{i}, e_{j}\right) .
$$

Thus:

$$
\begin{aligned}
I I_{0}(f)\left(e_{i}, e_{j}\right) & =I I_{0}^{e}(f)\left(e_{i}, e_{j}\right)+\left(I I_{0}(f)-I I_{0}^{e}(f)\right)\left(e_{i}, e_{j}\right) \\
& =\partial_{i} \partial_{j} f(0)+\delta_{i j} .
\end{aligned}
$$

In summary:

$$
I_{0}(f)=\left(\begin{array}{ll}
1 & 0 \\
0 & 1
\end{array}\right), \quad I I_{0}(f)=\left(\begin{array}{ll}
1+\lambda_{1} & 0 \\
0 & 1+\lambda_{2}
\end{array}\right), \quad I I I_{0}(f)=\left(\begin{array}{ll}
\left(1+\lambda_{1}\right)^{2} & 0 \\
0 & \left(1+\lambda_{2}\right)^{2}
\end{array}\right) .
$$

Using the formula for the Gaussian curvature of $\hat{f}$, we obtain:

$$
\left(1+\lambda_{1}\right)\left(1+\lambda_{2}\right)=k \text {. }
$$

Moreover, if $J$ is the conformal structure induced over $S$ by the Gauss lift at height $r$ of $\hat{f}$, then:

$$
J e_{1}=r\left(1+\lambda_{1}\right) e_{2}, \quad J e_{2}=-r\left(1+\lambda_{2}\right) e_{1} .
$$

We thus define $\left(e_{1}^{\prime}, e_{2}^{\prime}\right)$ by:

$$
e_{1}^{\prime}=\frac{1}{\sqrt{1+\lambda_{1}}} e_{1}, \quad e_{2}^{\prime}=\frac{1}{\sqrt{1+\lambda_{2}}} e_{2} .
$$

So that $J e_{1}^{\prime}=e_{2}^{\prime}$ and $J e_{2}^{\prime}=-e_{1}^{\prime}$. With respect to the basis $\left(e_{1}^{\prime}, e_{2}^{\prime}\right)$ in the domain, and the basis $\left(e_{1}, e_{2}\right)$ in the range, the matrix of $D\left(\vec{n} \circ \mathrm{N}_{\hat{f}}\right)$ is therefore equal to $M^{\prime}$, where:

$$
M^{\prime}=\frac{1}{2}\left(\begin{array}{ll}
\frac{2+\lambda_{1}}{\sqrt{1+\lambda_{1}}} & 0 \\
0 & \frac{2+\lambda_{2}}{\sqrt{1+\lambda_{2}}}
\end{array}\right)
$$


The complex dilatation of $M^{\prime}$ is equal to $\mu$, where:

$$
\mu=\frac{\left(\sqrt{1+\lambda_{1}}-\sqrt{1+\lambda_{2}}\right)}{\left(\sqrt{1+\lambda_{1}}+\sqrt{1+\lambda_{2}}\right)} \frac{r-1}{r+1} .
$$

The absolute value of the first multiplicand is trivially no greater than 1 , and so:

$$
|\mu| \leqslant \frac{r-1}{r+1} .
$$

$M^{\prime}$ is therefore $r$-quasiconformal, and this completes the proof.

\section{3 - Weak Compactness.}

We first discuss the form of degenerate limit that may arise. Let $\Gamma \subseteq \mathbb{H}^{3}$ be a complete geodesic in $\mathbb{H}^{3}$. Let $\mathrm{N}_{r}(\Gamma) \subseteq S_{r} \mathbb{H}^{3}$ be the set of vectors of length $r$ over $\Gamma$ which are normal to $\Gamma$.

\section{Definition 3.1}

Let $S$ be a surface and let $\hat{\imath}: S \rightarrow S_{r} \mathbb{H}^{3}$ be a complete immersion. We say that $(S, \hat{\imath})$ is tubular if and only if there exists a complete geodesic $\Gamma$ such that $\hat{\imath}$ is a covering map of $\mathrm{N}_{r}(\Gamma)$.

Remark: Observe that if $(S, \hat{\imath})$ is tubular, and if $J$ is the complex structure induced over $S$ by $\hat{\imath}$, then $(S, J)$ is of parabolic type. This plays an important role in the sequel.

We recall the definition of convergence modulo reparametrisation for complete immersed submanifolds. Let $(M, g)$ be a Riemannian manifold. For all $n \in \mathbb{N}$, let $i_{n}: S_{n} \rightarrow M$ be a complete immersion, and for all $n$, choose $p_{n} \in S_{n}$. Let $i_{0}: S_{0} \rightarrow M$ be another complete immersion and choose $p_{0} \in S_{0}$.

\section{Definition 3.2}

We say that $\left(S_{n}, i_{n}, p_{n}\right)_{n \in \mathbb{N}}$ converges to $\left(S_{0}, i_{0}, p_{0}\right)$ in the $C^{\infty}$ sense modulo reparametrisation if and only if there exists a sequence of mappings $\varphi_{n}: S_{0} \rightarrow S_{n}$ such that:

(i) for all $n, \varphi_{n}\left(p_{0}\right)=p_{n}$; and

for every relatively compact open subset $\Omega \subseteq S_{0}$, there exists $N \in \mathbb{N}$ such that:

(ii) for all $n \geqslant N$, the restriction of $\varphi_{n}$ to $\Omega$ is a diffeomorphism onto its image; and

(iii) $\left(i_{n} \circ \varphi_{n}\right)_{n \in \mathbb{N}}$ converges to $i_{0}$ in the $C_{\text {loc }}^{\infty}$ sense over $\Omega$.

We will refer to $\left(\varphi_{n}\right)_{n \in \mathbb{N}}$ as a sequence of convergence mappings for $\left(S_{n}, i_{n}, p_{n}\right)_{n \in \mathbb{N}}$ with respect to $\left(S_{0}, i_{0}, p_{0}\right)$.

Choose $k \in] 0,1\left[\right.$ and let $r=k^{-1 / 2}$. For $n \in \mathbb{N}$, let $S_{n}$ be an oriented surface, let $i_{n}: S_{n} \rightarrow$ $\mathbb{H}^{3}$ be a locally strictly convex immersion of constant Gaussian curvature equal to $k$, and let $p_{n}$ be a point in $S_{n}$. For all $n$, let $\hat{\imath}_{n, r}: S_{n} \rightarrow S_{r} \mathbb{H}^{3}$ be the Gauss lift of $i_{n}$ at height $r$. In [9], Labourie proves: 


\section{Theorem 3.3}

\section{Suppose that:}

(i) for all $n, \hat{\imath}_{n, r}: S \rightarrow S_{r} \mathbb{H}^{3}$ is a complete immersion; and

(ii) there exists a compact subset $K \subseteq S_{r} \mathbb{H}^{3}$ such that $\hat{\imath}_{n, r}\left(p_{n}\right) \in K$ for all $n$.

Then there exists a complete pointed immersed surface $\left(S_{0}, \hat{\imath}_{0}, p_{0}\right)$ towards which $\left(S_{n}, \hat{\imath}_{n}, p_{n}\right) \subseteq S_{r} \mathbb{H}^{3}$ subconverges in the $C^{\infty}$ sense modulo reparametrisation. Moreover either:

(i) $\pi \circ \hat{\imath}_{0}$ is an immersion, where $\pi: S_{r} \mathbb{H}^{3} \rightarrow \mathbb{H}^{3}$ is the canonical projection; or

(ii) $\left(S_{0}, \hat{\imath}_{0}\right)$ is tubular.

Remark: Observe that, in the first case, $\pi \circ \hat{\imath}_{0}$ is an immersion of constant Gaussian curvature equal to $k$. Importantly, it is not necessarily complete.

We now relate the convergence maps to the conformal structure of the limit. We require the following Schwartz lemma:

\section{Lemma 3.4}

Let $S$ be an oriented surface. Let $\left(J_{n}\right)_{n \in \mathbb{N}}$ be a sequence of smooth almost complex structures over $S$ which converges to another almost complex structure $J_{0}$ in the $C_{\text {loc }}^{\infty}$ sense. For all $n \in \mathbb{N}$, let $\alpha_{n}: S \rightarrow \mathbb{D}$ be holomorphic with respect to $J_{n}$. There exists $\alpha_{0}: S \rightarrow \mathbb{D}$ towards which $\left(\alpha_{n}\right)_{n \in \mathbb{N}}$ subconverges in the $C_{\text {loc }}^{\infty}$ sense.

Proof: Choose $p \in S$. Let $U$ be a neighbourhood of $p$, and for all $n \in \mathbb{N} \cup\{0\}$, let $\left(e_{1, n}, e_{2, n}\right)$ be a frame defined over $U$ such that:

(i) for all $n, J_{n} e_{1, n}=e_{2, n}$; and

(ii) $\left(e_{1, n}, e_{2, n}\right)_{n \in \mathbb{N}}$ converges to $\left(e_{1,0}, e_{2,0}\right)$ in the $C_{\text {loc }}^{\infty}$ sense.

For all $n \in \mathbb{N} \cup\{0\}$, define $a_{n}, b_{n}: \Omega \rightarrow \mathbb{R}$ such that:

$$
\left[e_{1, n}, e_{2, n}\right]=a_{n} e_{1, n}+b_{n} e_{2, n} .
$$

We define the generalised Laplacian $L_{n}$ such that, for all $f$ :

$$
L_{n} f=\left(e_{1, n} e_{1, n}+e_{2, n} e_{2, n}\right) f-\left(b_{n} e_{1, n}-a_{n} e_{2, n}\right) f .
$$

For all $n \in \mathbb{N}$, we denote $\alpha_{n}=\xi_{n}+i \eta_{n}$. The Cauchy-Riemann equations yield:

$$
\begin{aligned}
\left(e_{1, n} e_{1, n}+e_{2, n} e_{2, n}\right) \xi_{n} & =\left(e_{1, n} e_{2, n}-e_{2, n} e_{1, n}\right) \eta_{n} \\
& =\left(a_{n} e_{1, n}+b_{n} e_{2, n}\right) \eta_{n} \\
& =\left(b_{n} e_{1, n}-a_{n} e_{2, n}\right) \xi_{n} .
\end{aligned}
$$

Thus:

$$
L_{n} \xi_{n}=0
$$




\section{Hyperbolic Plateau problems}

For all $n$ :

$$
\left\|\xi_{n}\right\|_{L^{\infty}} \leqslant\left\|\alpha_{n}\right\|_{L^{\infty}} \leqslant 1
$$

It thus follows by classical elliptic regularity (c.f. [2]) that there exists $\xi_{0}$ towards which $\left(\xi_{n}\right)_{n \in \mathbb{N}}$ subconverges. Likewise, there exists $\eta_{0}$ towards which $\left(\eta_{n}\right)_{n \in \mathbb{N}}$ subconverges. This completes the proof.

We refine Labourie's result as follows: choose $k \in] 0,1\left[\right.$ and denote $r=k^{-1 / 2}$. Let $\mathbb{D}$ be the Poincaré disc. For all $n \in \mathbb{N}$, let $i_{n}: \mathbb{D} \rightarrow \mathbb{H}^{3}$ be a locally strictly convex immersion of constant Gaussian curvature equal to $k$, let $\hat{\imath}_{n, r}$ be its Gauss lift at height $r$. Suppose that $\hat{\imath}_{n, r}: \mathbb{D} \rightarrow S_{r} \mathbb{H}^{3}$ is complete, and, moreover, for all $n$, the complex structure induced over $\mathbb{D}$ by $\hat{\imath}_{n, r}$ coincides with the canonical complex structure of $\mathbb{D}$.

For all $n \in \mathbb{N}$, choose $z_{n} \in \mathbb{D}$. Let $K \subseteq S_{r} \mathbb{H}^{3}$ be compact, and suppose that $\hat{\imath}_{n, r}\left(z_{n}\right) \in K$. By Theorem 3.3, there exists a complete immersed surface $\left(S_{0}, \hat{\imath}_{0}, p_{0}\right)$ in $S_{r} \mathbb{H}^{3}$ towards which $\left(\mathbb{D}, \hat{\imath}_{n, r}, z_{n}\right)$ subconverges modulo reparametrisation in the $C_{\text {loc }}^{\infty}$ sense. Let $\left(\varphi_{n}\right)_{n \in \mathbb{N}}$ : $S_{0} \rightarrow \mathbb{D}$ be a sequence of convergence mappings of $\left(\mathbb{D}, \hat{\imath}_{n, r}, z_{n}\right)$ with respect to $\left(S_{0}, \hat{\imath}_{0}, p_{0}\right)$. Let $J_{0}$ be the complex structure induced over $S_{0}$ by $\hat{\imath}_{0}$.

\section{Lemma 3.5}

There exists a conformal mapping $\varphi_{0}:\left(S_{0}, J_{0}\right) \rightarrow \overline{\mathbb{D}}$ towards which $\left(\varphi_{n}\right)_{n \in \mathbb{N}}$ subconverges in the $C_{\text {loc }}^{\infty}$ sense.

Remark: Recall the remark following Definition 3.2. If $\left(S, J_{0}\right)$ is tubular, then, in particular it is of parabolic type, and so $\varphi_{0}$ is constant.

Proof: Let $\Omega \subseteq S_{0}$ be a relatively compact open set. Choose $N \in \mathbb{N}$ such that, for $n \geqslant N$, the restriction of $\varphi_{n}$ to $\Omega$ is a diffeomorphism onto its image. For all $n$, let $J_{n}$ be the complex structure induced over $S_{0}$ by $\left(\hat{\imath}_{n, r} \circ \varphi_{n}\right)$. Since $\left(\hat{\imath}_{n, r}\right)_{n \geqslant N}$ subconverges to $\hat{\imath}_{0}$ in the $C_{\mathrm{loc}}^{\infty}$ sense over $\Omega,\left(J_{n}\right)_{n \in \mathbb{N}}$ also subconverges to $J_{0}$ in the $C_{\mathrm{loc}}^{\infty}$ sense over $\Omega$. However, for all $n$, by definition $\varphi_{n}:\left(\Omega, J_{n}\right) \rightarrow \mathbb{D}$ is conformal. It follows by Lemma 3.4 that there exists a conformal map $\varphi_{0}:\left(\Omega, J_{0}\right) \rightarrow \mathbb{D}$ towards which $\left(\varphi_{n}\right)_{n \in \mathbb{N}}$ subconverges in the $C_{\text {loc }}^{\infty}$ sense. The result now follows by a diagonal argument.

\section{4 - Compactness of Quasiconformal Mappings.}

Let $\mathbb{D}$ be the Poincaré disc. We identify $\partial_{\infty} \mathbb{H}^{3}$, the ideal boundary of hyperbolic space, with the Riemann sphere, $\hat{\mathbb{C}}$. Let $\left(\varphi_{n}\right)_{n \in \mathbb{N}}, \varphi_{0}: \mathbb{D} \rightarrow \hat{\mathbb{C}}$ be locally conformal maps such that $\left(\varphi_{n}\right)_{n \in \mathbb{N}}$ converges to $\varphi_{0}$ in the $C_{\text {loc }}^{\infty}$ sense.

Choose $k \in] 0,1\left[\right.$ and denote $r=k^{-1 / 2}$. For all $n$, let $i_{n}: \mathbb{D} \rightarrow \mathbb{H}^{3}$ be an immersion of constant Gaussian curvature equal to $k$ such that:

(i) the Gauss lift, $\hat{\imath}_{n, r}$, of $i$ at height $r$ is a complete immersion of $\mathbb{D}$ into $S_{r} \mathbb{H}^{3}$; and

(ii) $\left(\vec{n} \circ \hat{\imath}_{n, r}\right)=\varphi_{n}$.

For all $n$, let $J_{n}$ be the complex structure induced over $\mathbb{D}$ by $\hat{\imath}_{n, r}$. Let $\mu_{n}$ be the complex dilatation of $J_{n}$ with respect to the canonical complex structure over $\mathbb{D}$. By Lemma 2.1, 
for all $n$ :

$$
\left\|\mu_{n}\right\| \leqslant \frac{r-1}{r+1} .
$$

\section{Proposition 4.1}

For all $n$, there exists a conformal homeomorphism $\alpha_{n}:\left(\mathbb{D}, J_{n}\right) \rightarrow \mathbb{D}$ such that $\alpha_{n}(0)=0$.

Proof: Since $J_{n}$ is $r$-quasiconformal with respect to the canonical complex structure over $\mathbb{D},\left(\mathbb{D}, J_{n}\right)$ is of hyperbolic type (c.f. [7]). The result follows by Riemann's uniformisation theorem.

\section{Proposition 4.2}

There exists a quasiconformal mapping $\alpha_{0}: \mathbb{D} \rightarrow \mathbb{D}$ such that $\alpha_{0}(0)=0$ and $\left(\alpha_{n}\right)_{n \in \mathbb{N}}$ subconverges to $\alpha_{0}$ in the $C^{0}$ sense.

Proof: We apply a Möbius transformation and work in the upper half space, $\mathbb{H}^{+}$. Choose $n \in \mathbb{N}$. Conjugating by a Möbius transformation, we may suppose that $\alpha_{n}$ is an $r$ quasiconformal homeomorphism from $\mathbb{H}^{+}$to itself which fixes $i$. We extend $\alpha_{n}$ to an $r$-quasiconformal homeomorphism of $\widehat{\mathbb{C}}$ as follows: let $\mu_{n}$ be the complex dilatation of $\alpha_{n}$. We extend $\mu_{n}$ uniquely to a bounded, measurable $(1,1)$-form over $\mathbb{C}$ such that, for all $z \in \mathbb{H}^{+}$:

$$
\mu_{n}(\bar{z})=\bar{\mu}_{n}(z) .
$$

By Theorem 4.25 of [7], there exists a unique quasiconformal homeomorphism $\tilde{\alpha}_{n}: \hat{\mathbb{C}} \rightarrow \hat{\mathbb{C}}$ preserving 0,1 and $+\infty$ and whose conformal dilatation is equal to $\mu_{n}$. Since its conformal dilatation and fixed points are symmetric about the real axis, $\tilde{\alpha}_{n}$ preserves $\mathbb{R}$. Since it fixes 0,1 and $\infty$, it also preserves the orientation of $\mathbb{R}$, and so, since it is orientation preserving, it maps $\mathbb{H}^{+}$to itself.

For all $n$, let $\beta_{n}: \hat{\mathbb{C}} \rightarrow \hat{\mathbb{C}}$ be a Möbius mapping also preserving $\mathbb{H}^{+}$such that:

$$
\beta_{n}\left(\tilde{\alpha}_{n}(i)\right)=i .
$$

Denote $\alpha_{n}^{\prime}=\beta_{n} \circ \tilde{\alpha}_{n}$. The mapping $\alpha_{n}$ is a homeomorphism of $\mathbb{H}^{+}$preserving $i$. Moreover, its conformal dilatation over $\mathbb{H}^{+}$is equal to that of $\tilde{\alpha}_{n}$, which is in turn equal to that of $\alpha_{n}$. It follows that, after composing $\beta_{n}$ with a rotation if necessary, $\alpha_{n}^{\prime}=\alpha_{n}$ over $\mathbb{H}^{+}$(c.f. [7]), and so $\alpha_{n}^{\prime}$ is the desired extension of $\alpha_{n}$. Henceforth, we denote $\alpha_{n}^{\prime}$ merely by $\alpha_{n}$. Observe that, for all $z \in \mathbb{H}^{+}$:

$$
\alpha_{n}(\bar{z})=\bar{\alpha}_{n}(z) .
$$

In particular, $\alpha_{n}$ preserves $i,-i$ and $\mathbb{R}$. Thus, by Theorem II.5.1 of [10], the sequence $\left(\alpha_{n}\right)_{n \in \mathbb{N}}$ constitutes a normal family, and, by Theorem II.5.3 of [10], it subconverges in the $C^{0}$ sense towards an $r$-quasiconformal mapping $\alpha_{0}: \hat{\mathbb{C}} \rightarrow \hat{\mathbb{C}}$ which preserves $i,-i$ and $\mathbb{R}$. This completes the proof.

\section{Proposition 4.3}

$\left(\alpha_{n}\right)_{n \in \mathbb{N}}^{-1}$ subconverges to $\alpha_{0}$ in the $C^{0}$ sense. 


\section{Hyperbolic Plateau problems}

Proof: As in the proof of Proposition 4.2, for all $n \in \mathbb{N} \cup\{0\}$, we extend $\alpha_{n}$ to an $r$ quasiconformal homeomorphism of $\hat{\mathbb{C}}$ to itself. Let $K$ and $\Omega$ be subsets of $\hat{\mathbb{C}}$ such that $K$ is compact, $\Omega$ is open, and $\alpha_{0}^{-1}(K) \subseteq \Omega$. The complements, $\Omega^{c}$ and $K^{c}$, are compact and open respectively. Bearing in mind that $\alpha_{0}$ is bijective:

$$
\begin{array}{rlrl} 
& \Omega^{c} & & \subseteq \alpha_{0}^{-1}(K)^{c} \\
& =\alpha_{0}^{-1}\left(K^{c}\right) \\
\Rightarrow \quad \alpha_{0}\left(\Omega^{c}\right) & \subseteq K^{c} .
\end{array}
$$

Since $\left(\alpha_{n}\right)_{n \in \mathbb{N}}$ converges uniformly to $\alpha_{0}$, there exists $N \in \mathbb{N}$ such that for $n \geqslant N$ :

$$
\begin{array}{rlrl} 
& \alpha_{n}\left(\Omega^{c}\right) & \subseteq K^{c} \\
\Rightarrow \quad \Omega^{c} & \subseteq \alpha_{n}^{-1}\left(K^{c}\right) \\
& & =\alpha_{n}^{-1}(K)^{c} \\
\Rightarrow \quad \alpha_{n}^{-1}(K) & \subseteq \Omega .
\end{array}
$$

The result follows by definition of uniform convergence.

\section{5 - Strong Compactness.}

We continue with the notation of the preceeding section. Let $\left(z_{n}\right)_{n \in \mathbb{N}} \in \mathbb{D}$ be a sequence in $\mathbb{D}$ converging to $z_{0}$ in $\mathbb{D}$. In order to apply Labourie's Theorem, we first show that $\left(i_{n}\left(z_{n}\right)\right)_{n \in \mathbb{N}}$ remains in a compact set. We achieve this in two steps:

\section{Proposition 5.1}

There exists a neighbourhood, $U$, of $\varphi_{0}\left(z_{0}\right)$ in $\mathbb{H}^{3} \cup \partial_{\infty} \mathbb{H}^{3}$ such that, for all $n$ :

$$
i_{n}\left(z_{n}\right) \in U^{c}
$$

Proof: For all $n \in \mathbb{N}$, let $\mathrm{N}_{n}$ be the normal vector field over $i_{n}$ compatible with the orientation, and define $I_{n}: \mathbb{D} \times\left[0, \infty\left[\rightarrow \mathbb{H}^{3}\right.\right.$ by:

$$
I_{n}(z, t)=\operatorname{Exp}\left(t \mathrm{~N}_{n}(z)\right)
$$

where Exp is the exponential map of $\mathbb{H}^{3}$. Let $B_{1}$ be the unit ball in $\mathbb{R}^{3}$. We identify $\mathbb{H}^{3}$ and $\partial_{\infty} \mathbb{H}^{3}$ with $B_{1}$ and $\partial B_{1}$ respectively in the canonical manner. For all $n, I_{n}$ extends to a smooth map from $\mathbb{D} \times[0, \infty]$ to $\bar{B}_{1}$ such that, for all $z \in \mathbb{D}$ :

$$
I_{n}(z, \infty)=\varphi_{n}(z)
$$

Let $V$ be a neighbourhood of $z_{0}$ in $\mathbb{D}$ and suppose that the restriction of $\varphi_{0}$ to $V$ is a diffeomorphism onto its image. Let $D \subseteq \partial B_{1}$ be a disc about $\varphi_{0}\left(z_{0}\right)$ in $\partial B_{1}$ which is contained in $\varphi_{0}(V)$. Without loss of generality, we may assume that, for all $n, z_{n} \in V$, the restriction of $\varphi_{n}$ to $V$ is a diffeomorphism onto its image, and $D \subseteq \varphi_{n}(V)$. 


\section{Hyperbolic Plateau problems}

Let $\Sigma_{0}$ be the totally geodesic hypersurface in $\mathbb{H}^{3}$ bounded by $\partial D$. For all $d>0$, let $\Sigma_{d}$ be the level hypersurface at distance $d$ from $\Sigma$ and let $j_{d}: \Sigma_{d} \rightarrow \mathbb{H}^{3}$ be the canonical embedding. Using elementary hyperbolic geometry, we show that, for all $d, \Sigma_{d}$ has constant Gauss curvature equal to $\tanh (d)^{2}$. Denote $d_{k}=\tanh ^{-1}(\sqrt{k})$. We claim that for all $n$, and for all $d \geqslant d_{k}, \Sigma_{d}$ lifts to an embedded surface in $\left.\mathbb{D} \times\right] 0,1[$. In other words, there exists $\tilde{\jmath}_{d, n}: \Sigma_{d} \rightarrow \mathbb{D} \times[0,1]$ such that:

(i) $I_{n} \circ \tilde{\jmath}_{d, n}=j_{d}$; and

(ii) the restriction of $\tilde{\jmath}_{d, n}$ to $\partial_{\infty} \Sigma_{d}=\partial D$ coincides with $\left(\left.\varphi_{n}\right|_{V}\right)^{-1}$.

Indeed, fix $n$. Let $j_{\infty}: D \rightarrow \partial_{\infty} \mathbb{H}^{3}$ be the canonical embedding. Trivially, $\left(\Sigma_{d}, j_{d}\right)$ converges to $\left(D, j_{\infty}\right)$ as $d$ tends to $+\infty$. For all $n \in \mathbb{N}$, we define $\tilde{\jmath}_{\infty, n}$ by:

$$
\tilde{\jmath}_{\infty, n}=\left(\left.\varphi_{n}\right|_{V}\right)^{-1} \circ j_{\infty}
$$

$\tilde{\jmath}_{\infty, n}$ is trivially a lift of $j_{\infty}$ satisfying both $(i)$ and $(i i)$. Since $I_{n}$ is everywhere a local diffeomorphism, for all sufficiently large $d, \tilde{\jmath}_{\infty, n}$ perturbs to a lift, $\tilde{\jmath}_{d, n}$ of $j_{d}$. Bearing in mind that $\hat{\imath}_{n}$ is complete, by continuously reducing $d, j_{d}$ can be lifted until we reach some $d=d_{0} \in[0, \infty]$ where $j_{d_{0}, n}$ meets $\mathbb{D} \times\{0\}$ at some point. Suppose that $d_{0}>d_{k}$. At the point of contact, $i_{n}$ is an interior tangent to $j_{d_{0}}$. However, the Gauss curvature of $\Sigma_{d}$ is greater than that of $i_{n}$, which is absurd by the geometric maximum principal. We deduce that $d_{0} \leqslant d_{k}$ and the assertion follows.

For sufficiently large $n, \varphi_{n}\left(z_{n}\right) \in D$, and so the geodesic ray in $\mathbb{H}^{3}$ leaving $i_{n}(\mathbb{D})$ at $z_{n}$ in the direction $\mathrm{N}_{n}\left(z_{n}\right)$ passes through $\Sigma_{d_{k}}$. However, this geodesic ray is also the geodesic segment in $\mathbb{H}^{3}$ joining $i_{n}\left(z_{n}\right)$ to $\varphi_{n}\left(z_{n}\right)$. We claim that there exists a neighbourhood, $U$, of $\varphi_{0}\left(z_{0}\right)$ such that, for sufficiently large $n$, and for any $p \in U$, the geodesic segment in $\mathbb{H}^{3}$ joining $p$ to $\varphi_{n}\left(z_{n}\right)$ does not intersect $\Sigma_{d_{k}}$. Indeed, suppose the contrary. There exists a sequence $\left(p_{n}\right)_{n \in \mathbb{N}} \in \mathbb{H}^{3}$ converging to $\varphi_{0}\left(z_{0}\right)$ such that, for all $n$, the geodesic segment in $\mathbb{H}^{3}$ joining $p_{n}$ to $\varphi_{n}\left(z_{n}\right)$ passes through $\Sigma_{d_{k}}$. However, these geodesic segments converge towards $\varphi_{0}\left(z_{0}\right)$ in the Haussdorf sense. Thus:

$$
\left\{\varphi_{0}\left(z_{0}\right)\right\} \cap \Sigma_{d_{k}} \neq \emptyset
$$

This is absurd, and the assertion follows. Thus, for sufficiently large $n, i_{n}\left(z_{n}\right) \notin U$. $U$ is therefore the desired neighbourhood of $\varphi_{0}\left(z_{0}\right)$, and this completes the proof.

\section{Proposition 5.2}

For any compact subset $K \subseteq \mathbb{D}$, there exists a compact subset $L \subseteq \mathbb{H}^{3}$ such that, for any $n \in \mathbb{N}$ :

$$
i_{n}(K) \subseteq L
$$

Proof: Suppose that contrary. Without loss of generality, there exists $\left(z_{n}\right)_{n \in \mathbb{N}} \in \mathbb{D}$ converging to $z_{0} \in \mathbb{D}$ such that $\left(i_{n}\left(z_{n}\right)\right)_{n \in \mathbb{N}}$ converges to some point $p_{0} \in \partial_{\infty} \mathbb{H}^{3}$. By Proposition 5.1, $p_{0} \neq \varphi_{0}\left(z_{0}\right)$. Choose $p_{0}^{\prime} \in \mathbb{H}^{3}$, and, for all $n$, let $\Phi_{n}: \mathbb{H}^{3} \rightarrow \mathbb{H}^{3}$ be an isometry such that $\Phi_{n}\left(p_{n}\right)=p_{0}^{\prime}$ and $\Phi_{n}\left(\varphi_{n}\left(z_{n}\right)\right)=\varphi_{0}\left(z_{0}\right)$. 


\section{Hyperbolic Plateau problems}

For all $n$, denote $j_{n}=\Phi_{n} \circ \varphi_{n}$ and let $\hat{\jmath}_{n, r}$ be the Gauss lift of $j_{n}$ at height $r$. For all $n, \hat{\jmath}_{n, r}\left(z_{n}\right)$ lies in the fibre over $p_{0}^{\prime}$ which is compact and so, by Theorem 3.3 , there exists a complete, pointed immersed surface $\left(S_{0}, \hat{\jmath}_{0}, q_{0}\right)$ towards which $\left(\mathbb{D}, \hat{\jmath}_{n}, z_{n}\right)$ subconverges in the $C_{\text {loc }}^{\infty}$ sense modulo reparametrisation. Let $\left(\psi_{n}\right)_{n \in \mathbb{N}}: S_{0} \rightarrow \mathbb{D}$ be a sequence of convergence mappings of $\left(\mathbb{D}, \hat{\jmath}_{n}, z_{n}\right)$ with respect to $\left(S_{0}, \hat{\jmath}_{0}, q_{0}\right)$. For all $n$, let $J_{n}$ be the complex structure induced over $\mathbb{D}$ by $\hat{\jmath}_{n}$. By Proposition 4.1 , for all $n$, there exists a conformal homeomorphism $\alpha_{n}:\left(\mathbb{D}, J_{n}\right) \rightarrow \mathbb{D}$ sending $z_{n}$ to 0. By Lemma 3.5, $\left(\alpha_{n} \circ \psi_{n}\right)_{n \in \mathbb{N}}$ converges in the $C_{\text {loc }}^{\infty}$ sense to a holomorphic mapping $\tilde{\psi}_{0}:\left(\Sigma_{0}, J_{0}\right) \rightarrow \mathbb{D}$ sending $q_{0}$ to 0 . Thus, by Proposition 4.3, $\left(\psi_{n}\right)_{n \in \mathbb{N}}$ converges in the $C_{\text {loc }}^{0}$ sense to $\psi_{0}:=\alpha_{0}^{-1} \circ \tilde{\psi}_{0}$. Trivially $\varphi_{0}\left(q_{0}\right)=z_{0}$.

Since $p_{0} \neq \varphi_{0}\left(z_{0}\right),\left(\Phi_{n}\right)_{n \in \mathbb{N}}$ converges locally uniformly over $\left(\mathbb{H}^{3} \cup \partial_{\infty} \mathbb{H}^{3}\right) \backslash\left\{p_{0}\right\}$ to the constant mapping sending every point to $\varphi_{0}\left(z_{0}\right)$. There thus exists a neighbourhood, $U$, of $z_{0}$ over which $\left(\Phi_{n} \circ \varphi_{n}\right)_{n \in \mathbb{N}}$ converges to a constant map, and so there exists a neighbourhood, $V$, of $q_{0}$ over which $\left(\Phi_{n} \circ \varphi_{n} \circ \psi_{n}\right)_{n \in \mathbb{N}}$ also converges to a constant map. However, for all $n$, bearing in mind that $\vec{n}$ commutes with $\Phi_{n}$ :

$$
\begin{aligned}
\Phi_{n} \circ \varphi_{n} \circ \psi_{n} & =\Phi_{n} \circ \vec{n} \circ \hat{\imath}_{n} \circ \psi_{n} \\
& =\vec{n} \circ \Phi_{n} \circ \hat{\imath}_{n} \circ \psi_{n} \\
& =\vec{n} \circ \hat{\jmath}_{n} \circ \psi_{n} .
\end{aligned}
$$

This converges in the $C_{\text {loc }}^{\infty}$ sense to $\vec{n} \circ \hat{\jmath}_{0}$ which is therefore constant over $V$. This is absurd, since, in both cases given in Theorem 3.3, $\vec{n} \circ \hat{\jmath}_{0}$ is a local homeomorphism. The result follows.

We are now in a position to prove:

\section{Proposition 5.3}

There exists a complete immersion $\hat{\imath}_{0}: \mathbb{D} \rightarrow S_{r} \mathbb{H}^{3}$ towards which $\left(\hat{\imath}_{n}\right)_{n \in \mathbb{N}}$ subconverges in the $C_{\text {loc }}^{\infty}$ sense. Moreover, $i_{0}=\pi \circ \hat{\imath}_{0}$ is an immersion.

Proof: For all $n$, let $A_{n}$ be the shape operator of $i_{n}$. We claim that for every compact subset $K \subseteq \mathbb{D}$, there exists $B>0$ such that for all $z \in K$, and for all $n,\left\|A_{n}(z)\right\| \leqslant B$. Indeed, suppose the contrary. Then, without loss of generality, there exists a sequence $\left(z_{n}\right)_{n \in \mathbb{N}} \in \mathbb{D}$ converging to a limit $z_{0} \in \mathbb{D}$ such that $\left(\left\|A_{n}(z)\right\|\right)_{n \in \mathbb{N}} \rightarrow+\infty$. By Proposition 5.2 , there exists a compact subset, $L \subseteq \mathbb{H}^{3}$ such that $i_{n}\left(z_{n}\right) \in L$ for all $n$. Thus, by Theorem 3.3 , there exists a complete, pointed immersion $\left(S_{0}, \hat{\imath}_{0}, p_{0}\right)$ towards which $\left(\mathbb{D}, \hat{\imath}_{n}, z_{n}\right)$ subconverges in the $C_{\text {loc }}^{\infty}$ sense modulo reparametrisation. Since $\left(\left\|A_{n}\right\|\right)_{n \in \mathbb{N}} \rightarrow+\infty, \pi \circ \hat{\imath}_{0}$ cannot be an immersion at $p_{0}$, and the immersed surface $\left(S_{0}, \hat{\imath}_{0}\right)$ is therefore tubular.

Let $\left(\psi_{n}\right)_{n \in \mathbb{N}}: S_{0} \rightarrow \mathbb{D}$ be a sequence of convergence mappings of $\left(\mathbb{D}, \hat{\imath}_{n}, z_{n}\right)_{n \in \mathbb{N}}$ with respect to $\left(S_{0}, \hat{\imath}_{0}, p_{0}\right)$. For all $n$, let $J_{n}$ be the complex structure induced over $\mathbb{D}$ by $\hat{\imath}_{n}$. By Proposition 4.1, for all $n$, there exists a conformal homoemorphism $\alpha_{n}:\left(\mathbb{D}, J_{n}\right) \rightarrow$ $\mathbb{D}$ sending $z_{n}$ to 0 . By Lemma 3.5, $\left(\alpha_{n} \circ \psi_{n}\right)_{n \in \mathbb{N}}$ subconverges in the $C_{\text {loc }}^{\infty}$ sense to a holomorphic mapping $\tilde{\psi}_{0}:\left(S_{0}, J_{0}\right) \rightarrow \mathbb{D}$ sending $q_{0}$ to 0 . Since $\left(S_{0}, \hat{\imath}_{0}\right)$ is tubular, in particular, it is of parabolic type, and so $\tilde{\psi}_{0}$ is constant. However, by Proposition 4.3, $\left(\psi_{n}\right)_{n \in \mathbb{N}}$ converges in the $C_{\text {loc }}^{0}$ sense to $\psi_{0}:=\alpha_{0}^{-1} \circ \tilde{\psi}_{0}$. Since $\tilde{\psi}_{0}$ is constant, so is $\psi_{0}$. 


\section{Hyperbolic Plateau problems}

Thus, in particular, $\left(\varphi_{n} \circ \psi_{n}\right)_{n \in \mathbb{N}}$ converges in the $C_{\mathrm{loc}}^{0}$ sense to a constant mapping. However, for all $n$ :

$$
\varphi_{n} \circ \psi_{n}=\vec{n} \circ \hat{\imath}_{n} \circ \psi_{n} .
$$

Since $\left(\hat{\imath}_{n} \circ \psi_{n}\right)_{n \in \mathbb{N}}$ converges in the $C_{\text {loc }}^{\infty}$ sense to $\hat{\imath}_{0}$, we conclude that $\vec{n} \circ \hat{\imath}_{0}$ is a constant mapping. This is absurd and the assertion follows.

By Theorem 3.3, there exists a complete, pointed immersion $\left(S_{0}, \hat{\imath}_{0}, p_{0}\right)$ towards which $\left(\mathbb{D}, \hat{\imath}_{n}, 0\right)$ subconverges in the $C^{\infty}$ sense modulo reparametrisation. Let $\left(\psi_{n}\right)_{n \in \mathbb{N}}: S_{0} \rightarrow \mathbb{D}$ be a sequence of convergence mappings of $\left(\mathbb{D}, \hat{\imath}_{n}, 0\right)$ with respect to $\left(S_{0}, \hat{\imath}_{0}, p_{0}\right)$. As before $\left(\psi_{n}\right)_{n \in \mathbb{N}}: S_{0} \rightarrow \mathbb{D}$ converges in the $C_{\text {loc }}^{0}$ sense to some $\psi_{0}: S_{0} \rightarrow \mathbb{D}$. Taking limits, we obtain:

$$
\varphi_{0} \circ \psi_{0}=\vec{n} \circ \hat{\imath}_{0}
$$

and so $\varphi_{0} \circ \psi_{0}$ is everywhere a local diffeomorphism. It follows that $\psi_{0}$ is a local diffeomorphism and $\left(\psi_{n}\right)_{n \in \mathbb{N}}$ subconverges to $\psi_{0}$ in the $C_{\text {loc }}^{\infty}$ sense.

We claim that $\psi_{0}$ is a covering map. Let $\gamma:[0,1] \rightarrow \mathbb{D}$ be a curve such that $\gamma(0)=0$. Let $\hat{g}$ be the Sasaki metric over $T \mathbb{H}^{3}$. For all $n$, let $l_{n}(\gamma)$ be the length of $\gamma$ with respect to $\hat{\imath}_{n}^{*} \hat{g}$. By Proposition 5.2, there exists a compact subset, $L \subseteq \mathbb{H}^{3}$ such that $i_{n}(\gamma([0,1])) \subseteq L$ for all $n$. Since, in addition, $\left(\varphi_{n}\right)_{n \in \mathbb{N}}$ converges and $\left(A_{n}\right)_{n \in \mathbb{N}}$ is uniformly bounded, we show that $\left(\hat{\imath}_{n} \circ \gamma\right)_{n \in \mathbb{N}}$ is uniformly bilipschitz. There thus exists $R>0$ such that, for all $n$ :

$$
l_{n}(\gamma) \leqslant R
$$

Thus, for sufficiently large $n, \gamma$ lifts through $\psi_{n}$ to a smooth curve $\gamma_{n} \subseteq S_{0}$ of length at most $2 R$. Taking limits, we see that $\gamma$ lifts through $\psi_{0}$ to a smooth curve in $S_{0}$, and the assertion follows.

Since $\psi_{0}$ is a covering map and $\mathbb{D}$ is simply connected, $\psi_{0}$ is a diffeomorphism. Let $K \subseteq \mathbb{D}$ be compact. Let $\Omega \subseteq \mathbb{D}$ be a relatively compact neighbourhood of $\mathbb{D}$. There exists $N \in \mathbb{N}$ such that, for all $n \geqslant N, \psi_{n}$ has a smooth inverse, $\beta_{n}: \Omega \rightarrow S_{0}$. Moreover, $\left(\beta_{n}\right)_{n \in \mathbb{N}}$ converges in the $C_{\text {loc }}^{\infty}$ sense to $\left(\left.\psi_{0}\right|_{\Omega}\right)^{-1}$. For all $n$ :

$$
\hat{\imath}_{n}=\hat{\imath}_{n} \circ \psi_{n} \circ \beta_{n}
$$

Thus $\left(\hat{\imath}_{n}\right)_{n \in \mathbb{N}}$ converges in the $C_{\text {loc }}^{\infty}$ sense to $\hat{\imath}_{0} \circ\left(\left.\psi_{0}\right|_{\Omega}\right)^{-1}$. This completes the proof.

Theorems 1.4 and 1.5 follow readily:

Proof of Theorem 1.4: For all $t<1$, define $\alpha_{t}: \mathbb{D} \rightarrow \mathbb{D}$ by:

$$
\alpha_{t}(z)=t z
$$

By Theorem $E$ of [9], for all $t$, there exists $i_{t}: \mathbb{D} \rightarrow \mathbb{H}^{3}$ such that:

(i) $i_{t}$ has constant Gaussian curvature equal to $k$;

(ii) $\hat{\imath}_{t, r}$ is complete; and 


\section{Hyperbolic Plateau problems}

(iii) $\vec{n} \circ \hat{\imath}_{t, r}=\varphi \circ \alpha_{t}$.

Letting $t$ converge to 1 , existence follows by Theorem 1.3. Uniqueness follows by Theorem $A$ of $[9]$. This completes the proof.

Proof of Theorem 1.5: Since $\varphi_{n}$ is conformal for all $n,\left(\varphi_{n}\right)_{n \in \mathbb{N}}$ converges to $\varphi_{0}$ in the $C_{\text {loc }}^{\infty}$ sense. Let $\left(i_{k_{n}}\right)_{n \in \mathbb{N}}$ be a subsequence of $\left(i_{n}\right)_{n \in \mathbb{N}}$. By Theorem 1.3, there exists $i_{0}^{\prime}$ towards which $\left(i_{k_{n}}\right)_{n \in \mathbb{N}}$ subconverges in the $C_{\text {loc }}^{\infty}$ sense such that:

(i) $i_{0}^{\prime}$ has constant Gaussian curvature equal to $k$;

(ii) $\left(\hat{\imath}_{0, r}^{\prime}\right)$ is complete; and

(iii) $\vec{n} \circ \hat{\imath}_{0, r}^{\prime}=\varphi_{0}$.

By uniqueness (c.f. Theorem $A$ of $[9]), i_{0}^{\prime}=i$. Thus, every subsequence of $\left(i_{n}\right)_{n \in \mathbb{N}}$ has a subsubsequence converging to $i_{0}$, and so $\left(i_{n}\right)_{n \in \mathbb{N}}$ itself converges to $i_{0}$. This completes the proof.

\section{6 - Bibliography.}

[1] Ballmann W., Gromov M., Schroeder V., Manifolds of nonpositive curvature, Progress in Mathematics, 61, Birkhäuser Boston Inc., Boston, MA, (1985)

[2] Brezis H., Functional analysis, Sobolev spaces and partial differential equations, Universitext, Springer, New York, (2011)

[3] Caffarelli L., Nirenberg L., Spruck J., Nonlinear second-order elliptic equations. V. The Dirichlet problem for Weingarten hypersurfaces, Comm. Pure Appl. Math. 41 (1988), no. 1, 47-70

[4] Gauss C. F., Disquisitiones generales circa superficies curvas, Oct. 81827

[5] Gromov M., Pseudoholomorphic curves in symplectic manifolds, Invent. Math. 82 (1985), no. 2, 307-347

[6] Guan B., Spruck J., The existence of hypersurfaces of constant Gauss curvature with prescribed boundary, J. Differential Geom. 62 (2002), no. 2, 259-287

[7] Imayoshi Y., Taniguchi M., An introduction to Teichmüller space, Springer-Verlag, Tokyo, (1992)

[8] Labourie F., Problème de Minkowski et surfaces à courbure constante dans les variétés hyperboliques, Bull. Soc. Math. France 119 (1991), no. 3, 307-325

[9] Labourie F., Un lemma de Morse pour les surfaces convexes, Invent. Math. 141 (2000), no. 2, 239-297

[10] Lehto O., Virtanen K. I., Quasiconformal mappings in the plane, die Grundlehren der mathematischen Wissenschaften, 126, Springer-Verlag, New-York, Heidelberg, (1973)

[11] Corro A. V., Martínez A., Milán F., Complete flat surfaces with two isolated singularities in hyperbolic 3-space, J. Math. Anal. Appl. 366 (2010), no. 2, 582-592 


\section{Hyperbolic Plateau problems}

[12] Pogorelov A. V., Extrinsic geometry of convex surfaces, Translations of Mathematical Monographs, Vol. 35. American Mathematical Society, Providence, R.I., (1973)

[13] Rosenberg H., Spruck J., On the existence of convex hypersurfaces of constant Gauss curvature in hyperbolic space, J. Differential Geom. 40 (1994), no. 2, 379-409

[14] Sasaki S., On the differential geometry of tangent bundles of Riemannian manifolds, Tôhoku Math. J. (2) 10 (1958), 338-354

[15] Smith G., Pointed k-surfaces, Bull. Soc. Math. France 134, no. 4, (2006), 509-557

[16] Smith G., Moduli of Flat Conformal Structures of Hyperbolic Type, to appear in Geom. Dedicata

[17] Smith G., Compactness for immersions of prescribed Gaussian curvature II - geometric aspects, arXiv:1002.2982

[18] Trudinger N. S., Wang X. J., On locally convex hypersurfaces with boundary, J. Reine Angew. Math. 551 (2002), 11-32 